\title{
Écosystème
}

\section{LA MUSIQUE, TREMPLIN DU CORPS DANSANT}

\section{Claudia Blouin}

Volume 2, numéro 1, 2019

Danse

URI : https://id.erudit.org/iderudit/1062558ar

DOI : https://doi.org/10.7202/1062558ar

Aller au sommaire du numéro

Éditeur(s)

La chambre blanche

ISSN

2562-3222 (numérique)

Découvrir la revue

Citer cet article

Blouin, C. (2019). LA MUSIQUE, TREMPLIN DU CORPS DANSANT. Écosystème,

2(1), 9-14. https://doi.org/10.7202/1062558ar d'utilisation que vous pouvez consulter en ligne.

https://apropos.erudit.org/fr/usagers/politique-dutilisation/ 


\title{
LA MUSIQUE, TREMPLIN DU CORPS DANSANT
}

\section{Claudia Blouin}

\author{
Comment alors faire léviter le corps des \\ vivants pour l'extirper du cloaque? \\ Il existe un moyen pour cela : la musique ${ }^{1}$.
}

— Satoshi Miyagi

Dans sa mise en scène de Révélation Red in Blue trilogie, présentée au Théâtre de la Colline à Paris à l'automne 2018, Satoshi Miyagi prête à la musique le pouvoir de retirer à ses interprètes leur gravité terrestre et humaine. Au son des marimbas et des tambours, ces derniers sont propulsés dans le monde des âmes qui voyagent entre la vie et la mort. Ainsi, les acteurs-danseurs-musiciens entraînent avec eux les spectateurs dans l'univers symbolique du texte de Léonora Miano. Cette capacité de la musique à soulever et transporter autant les interprètes que le public ouvre la réflexion sur son influence au sein des arts de la scène et plus particulièrement sur la danse, avec laquelle elle entretient une relation privilégiée. Dans le cadre d'une maîtrise de recherche et création à l'Université Laval, j'ai exploré l'hypothèse selon laquelle la musique possède un potentiel théâtral et dramaturgique à exploiter dans le processus de création chorégraphique ${ }^{2}$. L'utilisation d'œuvres instrumentales comme inspiration pour des scénarios et comme trame structurant des séquences de danse théâtralisée m'a permis de comprendre un peu mieux comment la musique agit sur le corps dansant. On peut dire qu'elle travaille autant sur l'espace dans lequel il évolue que directement sur le corps lui-même. Elle stimule ses capacités expressives. Plus que tout, la musique est une partenaire sur laquelle le danseur peut s'appuyer. Il a la possibilité d'entrer en dialogue avec elle à travers la structure temporelle qu'ils sont amenés à partager.

\section{Ouvrir l'espace}

Tout comme le corps, le son est une matière qui traverse l'espace de la scène. Il l'habite de manière à transformer le lieu, à y insuffler des atmosphères. Dans $A$ Choreographer's Handbook, Jonathan Burrows souligne le potentiel de ce principe : " It is easy to create an atmosphere using sound or music. The atmosphere created by sound or music can give the performance a sense of greater meaning, carried by the emotional landscape of what we hear $»^{3}$. Les sons peuvent rappeler des objets, certaines musiques peuvent évoquer une époque, un lieu géographique particulier. C'est le cas dans Révélation de Miyagi, où la musique percussive réussit à suggérer l'environnement subsaharien dont il est question dans le texte, et ce, au sein d'une production principalement japonaise dans son esthétique visuelle. Dans mes propres laboratoires, l'utilisation des pièces jazz de Duke Ellington

1 Satoshi Miyagi, «S'adresser aux âmes des morts et des vivants », Révélation Red in Blue trilogie, Paris, La Colline Théâtre National, 2018, f. 10.

2 Claudia Blouin, Le potentiel théâtral et dramaturgique de la musique dans le processus de création chorégraphique, mémoire de maîtrise en Littérature et arts de la scène et de l'écran, Québec, Université Laval, 2017, $161 \mathrm{p}$.

3 Jonathan Burrows, A Choreographer's Handbook, New York, Routledge, 2010, p. 183. 
amenait une américanité au traitement chorégraphique que j'en ai fait, notamment par une esthétique inspirée du cinéma muet. Ce serait donc que la musique trace dans l'espace un paysage où les danseurs sont invités à évoluer. Il est même possible d'y voir - plutôt d'y entendre ! - un décor sonore.

Dans son ouvrage Dramaturgy of Sound in the Avant-garde and Postdramatic Theater, Mladen Ovadija traite de l'importance de la qualité matérielle et concrète du son comme élément constitutif du spectacle vivant, au même titre que la lumière et les constructions scénographiques. Des expérimentations de John Cage et Marcel Duchamp autour de l'idée d'une sculpture musicale faite de sons enveloppant l'auditeur, elle déduit: "sound is capable of producing the "virtual volume » of a sculpture " ${ }^{4}$. Selon cette proposition, le son et la musique ont le pouvoir de dévoiler des formes multidimensionnelles. Cela implique, pour la danse, une dynamisation de l'espace où s'exprime le mouvement. L'interprète en danse peut choisir de travailler de concert avec le matériel qui s'offre à son ouïe. Les volumes sonores qui traversent son terrain d'exploration le transportent dans un environnement aux paramètres sans cesse renouvelés. Ils modulent ses déplacements et son tracé dans l'espace.

Burrows met par ailleurs en garde le lecteur de son guide du chorégraphe contre ce pouvoir évocateur et transformateur de la musique. Le poids des atmosphères qu'elle dévoile peut écraser une atmosphère plus subtile que la danse en elle-même chercherait à transmettre. L'espace ouvert par la musique ne se limite pas à la scène et aux interprètes. Le spectateur est baigné dans la conception sonore d'une performance. Il existe donc un risque qu'il ait plus difficilement accès à ce qu'exprime le corps dansant. Sa connexion affective avec la musique prend par moments le dessus sur une possible connexion visuelle et kinesthésique avec la danse. C'est pourquoi certains créateurs choisissent de limiter le volume de la diffusion sonore, parfois pour l'oreille seule des interprètes. Le concepteur sonore français Mathieu Doze, dans son travail avec la chorégraphe Emmanuelle Huynh, pratique ce type d'approche :

Toute la partie centrale de Tôzai !... est une trame de sons qui s'enchâssent. Ils sont à la limite du subliminal en termes de niveau de diffusion. Je les agence à l'oreille, sachant d'où et quand ils vont sortir, ce qui me permet de jouer sur de "l'à peine", de fabriquer comme une brume sonore à laquelle j'espère que les oreilles des autres s'habitueront, exactement comme l'œil finit par se repérer dans le noir ${ }^{5}$.

Sa conception sonore en direct participe à nourrir les danseurs et à leur ouvrir un horizon partagé. Puisque Doze use de subtilité et de discrétion, sa musique n'écrase pas la danse en stimulant excessivement le spectateur. Elle installe plutôt une atmosphère composée de sons liés à la thématique du spectacle. Lorsque ces petits volumes traversent la scène et

4 Mladen Ovadija, Dramaturgy of Sound in the Avant-garde and Postdramatic Theater, Montréal et Kingston, McGillQueen's University Press, 2013, p. 144.

5 Jean-Paul Quiennec, « Émancipation et pluralité des pratiques sonores », L'Annuaire Théâtral, no. 56-57, 2014-2015, p. 247. 
croisent le chemin des danseurs, ils concourent au déploiement du mouvement. C'est en ce sens qu'on peut parler d'un tremplin pour le corps dansant.

\section{Transformer le corps}

Si la musique habite et agit sur l'espace qui entoure le danseur, elle pénètre également son corps et affecte, de l'intérieur, la qualité de son mouvement. L'expérience musicale ne se restreint pas à l'oreille et à l'interprétation cérébrale des stimuli. Mladen Ovadija cite le post-phénoménologue Don Idhe pour expliquer l'aspect immersif et incarné de l'écoute :

Phenomenologically I do not merely hear with my ears, I hear with my whole body... This may be detected quite dramatically in listening to loud rock music. The bass notes reverberate in my stomach, and even my feet «hear» the sound of the auditory orgy... The bodily involvement comprises the range from soothing pleasure to the point of insanity in the continuum of possible sound in music and noise ${ }^{6}$.

La musique produit des réactions physiques directes et profondes sur le corps humain. Elle est ressentie jusque dans les entrailles. Elle peut apaiser ou même provoquer de la douleur. L'interprète en danse qui travaille avec du matériel musical est exposé à ces réactions affectives du corps. Le jeu de contraction et de détente de ses muscles, l'amplitude, le rythme et la vitesse de ses gestes seront empreints des effets de la musique sur son état intérieur.

Tel un costume que l'on enfile en soi, la musique travestit le corps dansant. Elle participe à la construction d'une figure que l'interprète donne à voir sur la scène et qui n'est pas tout à fait lui-même. Le plasticien allemand Oskar Schlemmer, s'intéressant aux dimensions formelles du théâtre et de la danse, travaillait le costume comme une contrainte qui serait source d'expressivité. L'impact du costume sur le corps de l'interprète devait modifier son attitude, sa posture, sa démarche et donc influencer son expression.

L'acteur peut alors être changé, transformé, ensorcelé par tout objet, masque, costume, accessoire qui lui est adjoint au point que le comportement, que la structure du corps et de l'esprit de celui qui les porte en soit déséquilibré, ou plutôt équilibrée d'une autre façon (la nature de l'acteur et des gens-qui-sont-en-représentation se révèle le plus clairement à la mesure des modifications de leur comportement par ce genre d'appendices qui leur sont adjoints, que ce soit une cigarette, un chapeau, une canne, un vêtement, etc) ${ }^{7}$.

Dans les Ballets triadiques de Schlemmer, les tissus et objets qui habillent les interprètes entravent leurs gestes. Ils leur suggèrent tantôt un rythme, tantôt une asymétrie. Bref, ils

\footnotetext{
6 Don Ihde, Listening and Voice : A Phenomenology of Sound, Athens, Ohio University Press, 1976, p. 45.

7 Oskar Schlemmer, Théâtre et abstraction : l'espace du Bauhaus, Lausanne, L'Âge d'homme, 1978, p. 50.
} 
modifient leur manière d'être en scène. Les sons auxquels nous confrontons les danseurs, de par leur qualité vibratoire, donc matérielle, possèdent ce même potentiel de transformation. Dans le cadre de mon projet de recherche-création à la maîtrise, j'ai expérimenté la construction de figures dramatiques associées à différents instruments musicaux. Ainsi, un interprète invité à explorer par improvisation la tonalité et la rythmique du marimba dans la pièce The Enchanted Dawn de Ravi Shankar proposait une qualité de mouvement saccadée et sautillante. Ces caractéristiques de sa danse participaient à esquisser les traits d'un personnage nerveux. On peut dire que la musique a influencé la forme de ce dernier. Cette expérience, inspirée du formalisme de Schlemmer, souligne le pouvoir de la musique à déplacer l'identité que l'interprète dansant donne à voir sur la scène, à guider et à amplifier son expressivité.

\section{Dialoguer}

Outre sa tendance à moduler l'espace et le corps même du danseur, la musique s'offre également à lui comme une partenaire avec laquelle il est invité à entrer en dialogue. Favoriser une réciprocité dans la relation entre les deux disciplines ouvre la voie à des échanges stimulants. L'artiste multidisciplinaire Guy-André Lagesse, par exemple, caractérise ainsi son approche : «Ma préoccupation essentielle est de trouver comment rendre la musique spectaculaire, comment insuffler de la dynamique au rapport danseursmusiciens, en utilisant la musique comme un personnage $»{ }^{8}$. L'idée est de souligner le rôle actif de la musique dans la performance dansée, sa présence et sa participation à l'œuvre à titre de figure quasi anthropomorphique. Dans le cas d'improvisations impliquant musiciens et danseurs, le mouvement se nourrit du son et le son du mouvement. Cela crée une communication entre les plans visuels et auditifs de la performance. À la manière du jazz où les instrumentistes se répondent l'un à l'autre, danseurs et musiciens, chacun avec son langage propre, amorcent une conversation qui devient le noyau de l'œuvre.

Dans son article Dancing in the Imagined Space of Music, Rachel Duerden analyse les variations de cette forme de communication entre la musique et la danse. Elle affirme dans une de ses conclusions, " [i]t could be argued that much of the pleasure that we derive from dance-music dialogues stems from this play of convergences (consonances) and divergences (dissonances) $\gg{ }^{9}$. Selon elle, les moments de concordance entre le son et le mouvement apportent un sentiment de plénitude au spectateur tandis que les moments de divergence dynamisent le dialogue entre les deux médias. Les caractéristiques partagées comme le rythme, le tempo et la structure inscrite dans une temporalité définie peuvent être exploitées en contrepoint, en décalage ou en contraste. Ce jeu sur les variations participe à la construction d'une œuvre multidimensionnelle. Comme dans toute discussion, l'intérêt de l'échange entre corps musical et corps dansant vient autant, sinon plus, des oppositions et dissensions constructives que d'un accord mutuel et constant.

Une conversation s'engage lorsque le danseur répond par son mouvement à la musique qui ébranle son corps et sculpte son espace. Le public tient lieu de témoin dans cet échange. Si

8 Colloque Montpellier Danse, Création musicale et création chorégraphique : actes du Colloque Montpellier Danse, 9-10 juillet 1987, Paris, Le Centre, 1988, 65 p.

9 Rachel Duerden, « Dancing in the Imagined Space of Music », Dance Research : The Journal of the Society for Dance Research, vol. 25, no. 1, 2007, p. 74. 
l'on reprend l'idée que l'écoute de l'environnement sonore permet d'expérimenter une connivence ontologique entre les êtres et les choses - introduite par Vincent BouchardValentine dans le premier numéro d'Écosystème ${ }^{10}$ — on en vient à la conclusion que la musique favorise l'identification kinesthésique du spectateur. Elle l'implique dans la performance en faisant résonner l'espace et les corps. Agissant comme un tremplin, la musique réduit la distance qui sépare le corps dansant de son public.

10 Vincent Bouchard-Valentine, «Environnement, éducation et arts sonores : éléments d'ancrage théoriques pour un projet éducatif écosocial », Écosystème, vol. 1, 2018, pp. 9-19. 


\section{Références}

« Révélation Red in Blue Trilogie », Paris, La Colline Théâtre National, 2018. 17 f.

BLOUIN, Claudia. Le potentiel théâtral et dramaturgique de la musique dans le processus de création chorégraphique. Mémoire de maîtrise en Littérature et arts de la scène et de l'écran, Québec, Université Laval, 2017. 161 p.

BURROWS, Jonathan. A Choreographer's Handbook. New York, Routledge, 2010. 224 p.

Colloque MontPellier Danse. Création musicale et création chorégraphique : actes du Colloque Montpellier Danse, 9-10 juillet 1987. Paris, Le Centre, 1988. 65 p.

DUERDEN, Rachel. « Dancing in the Imagined Space of Music », dans Dance Research : The Journal of the Society for Dance Research. vol. 25, no.1, 2007. pp.73-83.

IHDE, Don. Listening and Voice : A Phenomenology of Sound. Athens, Ohio University Press, 1976. 296 p.

OVADIJA, Mladen. Dramaturgy of Sound in the Avant-garde and Postdramatic Theater. Montréal et Kingston, McGill-Queen's University Press, 2013. 252 p.

QUIENNEC, Jean-Paul. «Émancipation et pluralité des pratiques sonores », dans L'Annuaire Théâtral. no. 56-57, 2014-2015. pp. 229-253.

SCHLEMMER, Oskar. Théâtre et abstraction : l'espace du Bauhaus. Lausanne, L'Âge d'homme, 1978. $160 \mathrm{p}$. 